\title{
Evidence that high-migration drug-surviving MOLT4 leukemia cells exhibit cancer stem cell-like properties
}

\author{
XIAOXING HUANG $^{1 *}$, MENG XIONG $^{1 *}$, YUJIE JIN $^{1,4^{*}}$, CHAOHUA DENG $^{1}$, HUI XU $^{1}$, \\ CHANGQING AN ${ }^{2}$, LING HAO ${ }^{1}$, XIANGYONG YANG ${ }^{3}$, XINZHOU DENG ${ }^{1}$, \\ ZHENBO TU $^{1}$, XINRAN LI $^{1}$, RUIJING XIAO ${ }^{1}$ and QIUPING ZHANG ${ }^{1,5}$ \\ ${ }^{1}$ Department of Immunology, School of Basic Medical Sciences, Wuhan University, Wuhan, \\ Hubei 430071; ${ }^{2}$ Department of Pediatrics, Maternal and Child Health Hospital of Hubei Province; \\ ${ }^{3}$ Hubei University of Technology Engineering and Technology College, Wuhan, Hubei 430000; \\ ${ }^{4}$ Department of Clinical Laboratory, Taihe Hospital, Hubei University of Medicine, Shiyan City, Hubei 442000; \\ ${ }^{5}$ Hubei Provincial Key Laboratory of Developmentally Originated Disease, Wuhan, Hubei 430071, P.R. China
}

Received March 6, 2016; Accepted April 28, 2016

DOI: 10.3892/ijo.2016.3526

\begin{abstract}
Leukemia represents a spectrum of hematological malignancies threatening human health. Resistance to treatments and metastasis of leukemia are the main causes of death in patients. Leukemia stem cells (LSCs) are the initiating cells of leukemia as well as the main source of drug resistance, invasion and metastasis. Consequently, eliminating LSCs is a prerequisite to eradicate leukemia. Preliminary studies in our laboratory have shown that chemokines and their related receptors play an important role in the drug resistance and metastasis of leukemic cells. In this study, we obtained high migration drug-surviving (short term) MOLT4 cells (hMDSCs-MOLT4) with treatment of doxorubicin (DOX) after Transwell assay. Then we detected stem cellassociated molecular markers on hMDSCs-MOLT4 cells and the parental MOLT4 cells by FCM, QPCR, western blotting, H\&E staining and immunohistochemistry experimental techniques in vitro and in vivo. Moreover, we explored its impact
\end{abstract}

Correspondence to: Professor Qiuping Zhang or Dr Ruijing Xiao, Department of Immunology, School of Basic Medical Sciences, Wuhan University, 185 Donghu Road, Wuchang, Wuhan, Hubei 430071, P.R. China

E-mail: qpzhang@whu.edu.cn

E-mail: xrj7619@aliyun.com

*Contributed equally

Abbreviation: CSCs, cancer stem cells; CXCR4, CXC chemokine receptor 4; DOX, doxorubicin; DSCs, drug-surviving cells; hMDSCsMOLT4, high migration drug-surviving (short-term) MOLT4 cells; IOD, integrated optical density; IVC, individual ventilated cages; LSCs, leukemia stem cells; P-gp, P-glycoprotein

Key words: MOLT4 cells, drug survival cells, high migration, hMDSCs-MOLT4 cells, stem cell-like properties, drug resistance on drug resistance and tumor formation. Then we found that compared with the parental MOLT4 cells, the mRNA expression levels of stem cell-related factors Sox2, Oct4, C-myc, Klf4, Nanog, Bmi-1, CXCR4 are increased in hMDSCsMOLT4 cells, together with the protein expression levels of Sox2, Oct4, Klf4, Nanog, CXCR4 and CD34. Our results indicated that hMDSCs-MOLT4 cells exhibited strong drug resistance and certain cancer stem cell-like characteristics. It is the first indication that the targeting stemness factors such as Sox2, Oct4, Klf4, Nanog and CXCR4 may represent plausible options for eliminating T-ALL stem-like cells. The present findings shed light on the relationship between drugtolerant leukemic cells and cancer stem cells.

\section{Introduction}

The major causes of treatment failure for patients with leukemia are drug resistance and metastasis and traditional therapies cannot eradicate all aggressive leukemia cells. New chemotherapy drugs and hematopoietic stem cell transplantation technology have improved the remission and disease-free survival rate of leukemia patients, but still fail to prevent drug resistance and metastasis (1). One generally accepted theory is that leukemia is maintained by leukemia stem cells (LSCs) (2), which are quiescent and do not respond well to cell cycle-specific cytotoxic agents used to treat leukemia. It is therefore impossible to eradicate leukemia unless LSCs were eliminated $(3,4)$.

Cancer stem cells (CSCs) are tumor-initiating and tumorpropagating cells with enhanced resistance to chemotherapeutic drugs. Vice versa, tumor cells resistant to chemotherapeutic drugs have been shown to display certain phenotypes and characteristics of CSCs (5). For example, colon cancer cells treated with chemotherapy drugs exhibit CSC phenotypes (6). It is therefore speculated that drug-tolerant or drug-surviving tumor cells may be enriched in CSCs. In recent years, some CSC properties such as multidrug resistance $(7,8)$ and high migration ability have been employed to isolate CSCs (9). 
Theoretically, such strategies for investigating LSCs may well be worth the efforts since drug resistance and metastasis are very common and represent the worst clinical outcome in leukemia patients.

Some studies suggest that the phenotype of LSCs is CD34 ${ }^{+}$CD38- (10,11). Sox2, Oct4, Nanog, C-myc and Klf4 play essential roles in stem cell maintenance (12-14). Research from the Tang laboratory $(14,15)$ has demonstrated that Nanog may also play an important role in the self-renewal of CSCs. Lessard et al (16) have reported that Bmi-1 is a key factor in maintaining the self-renewal of LSCs although it is not required for the initial leukemia development.

Both CXC chemokine receptor 4 (CXCR4) and cell adhesion ligand receptor CD44 have been implicated in leukemia recurrence and metastasis. CXCR4 regulates tumor cell homing and migration (17) and has been implicated in maintaining prostate cancer stem-like cells and in the drug resistance of breast cancer stem-like cells to tamoxifen $(18,19)$. Moreover, CXCR4 has become a new target for the treatment of LSCs (20). On the other hand, the role of CD44 in LSCs seems to be controversial. Some studies suggest that CD44 contributes to the drug-resistance of LSCs to chemotherapeutics and promotes leukemia development (21), consistent with the reported involvement of CD44 in maintaining the stemness characteristics and tumor metastasis of prostate CSCs (22). On the other hand, there are also studies suggesting that CD44 (23) may also inhibit growth of tumor cells. Therefore, it will be worthwhile to explore the functions of CXCR4 and CD44 in the invasion and metastasis of leukemic cells or LSCs.

The aim of this study is to investigate the biological properties of chemotherapy-resistant leukemic cells with an ultimate goal of developing novel therapeutic strategies against the relapse of leukemia. Through our experiments we demonstrated that hMDSCs-MOLT4 cells possess many CSC-like phenotypes and properties.

\section{Materials and methods}

Cell lines and cell cultures. Human leukemia cell lines K562 (Chronic Myelogenous Leukemia cell line), HL60 (Acute Promyelocytic Leukemia cell line), MOLT4 (T-cell acute lymphocytic leukemia cell line, T-ALL) were obtained from ATCC and cultured in RPMI-1640 medium (Hyclone, Logan, UT, USA) supplemented with 10\% FBS (Life Technologies, Grand Island, NY, USA), penicillin (100 U/ml) and streptomycin $(100 \mu \mathrm{g} / \mathrm{ml})$. All cells were cultured in a humidified incubator at $37^{\circ} \mathrm{C}$ with $5 \% \mathrm{CO}_{2}$. Only cells in the logarithmic phase of growth were used for experiments.

MTT assays. Cells re-suspended in RPMI-1640 supplemented with $10 \%$ FBS were added into 96-well plates at 5000 cells/well in $100 \mu \mathrm{l}$ medium. Doxorubicin (DOX) was then added to cells at final concentrations of $0.05,0.1,0.2,0.4,0.6,0.8,1.0,1.2$, $1.4,1.6$, and $1.8 \mu \mathrm{g} / \mathrm{ml}$. Each drug concentration was set up in triplicate wells with no-drug wells as controls. After incubation at $37^{\circ} \mathrm{C}$ with $5 \% \mathrm{CO}_{2}$ for $24 \mathrm{~h}, 10 \mu \mathrm{l}$ MT solutions were added to each well for additional $4 \mathrm{~h}$. Cells in the plates were centrifuged at $1000 \mathrm{rpm}$ for $20 \mathrm{~min}$ and then $100 \mu \mathrm{l}$ DMSO was added to each well to dissolve any precipitate. Finally, cells were placed on a shaker at low speed for $20 \mathrm{~min}$ to fully dissolve the crystals and absorbance values were obtained by reading at $\mathrm{OD}_{570}$ on a micro-plate reader.

Establishment of drug-surviving cells (DSCs). HL60, K562, or MOLT4 cells were treated with DOX at the concentrations of $1.05 \mu \mathrm{g} / \mathrm{ml}, 1.35 \mu \mathrm{g} / \mathrm{ml}$, or $0.6 \mu \mathrm{g} / \mathrm{ml}$, respectively, for $48 \mathrm{~h}$, according to the $\mathrm{IC}_{50}$ concentrations of DOX on these cells. The surviving cells were termed DSCs and used for subsequent experiments.

Establishment of high migration drug-surviving (short-term) MOLT4 cells (hMDSCs-MOLT4). Briefly, $500 \mu$ l culture medium with $20 \%$ FBS was added in 24-well plate lower chamber, and $100 \mu \mathrm{l}$ MOLT4 cell suspension $\left(5 \times 10^{5} / \mathrm{ml}\right)$ added in the Transwell chambers. The pore size of Transwell is $0.8 \mu \mathrm{m}$. Cells were incubated at $37^{\circ} \mathrm{C}$ with $5 \% \mathrm{CO}_{2}$ for $24 \mathrm{~h}$. Then the chemotherapeutic drug DOX at $0.6 \mu \mathrm{g} / \mathrm{ml}$ was added to the lower chambers for $72 \mathrm{~h}$. The surviving cells were termed hMDSCs-MOLT4 and used for subsequent experiments. The percentage of imputs is $\sim 18.3 \%$.

Immunofluorescence. Cells were plated and cultured on sterilized polylysine-coated coverslips for $15 \mathrm{~min}$ followed by washing in PBS. To label the CXCR4 protein, cells were first fixed in 4\% paraformaldehyde in PBS for $10 \mathrm{~min}$, permeabilized in $0.1 \%$ Triton $\mathrm{X}-100$ for $3 \mathrm{~min}$ at room temperature, followed by incubation with an anti-CXCR4 antibody at $4{ }^{\circ} \mathrm{C}$ overnight. After washing in PBS, samples were incubated with an FITC-linked secondary antibody in the dark at room temperature for $30 \mathrm{~min}$ and then glass slides mounted in $90 \%$ glycerol. Images were visualized and acquired with an epifluorescence microscope.

Flow cytometry. Cells were harvested through centrifugation and washed once with cold PBS. Then the cells were incubated with FITC-anti-CD34, FITC-anti-CD38, PE-anti-CD44, PE-anti-CXCR4 (R\&D Systems; Minneapolis, MN, USA), or PE-anti-P-gp (eBioscience, San Diego, CA, USA) antibodies in the dark at $4^{\circ} \mathrm{C}$ for $30 \mathrm{~min}$, followed by washing in ice-cold PBS and finally re-suspended in $500 \mu \mathrm{l}$ PBS for flow cytometric analysis (Beckman, Miami, FL, USA). As control, cells were stained with the matching isotype control Abs.

RNA preparation, reverse transcriptase-polymerase chain reaction $(R T-P C R)$ and real-time quantitative PCR. Total RNA was isolated from MOLT4 cells and hMDSCs-MOLT4 using TRIzol reagent (Invitrogen, Merelbeke, Belgium) according to the manufacturer's instructions. The concentration and purity of total RNA were determined using spectrophotometry. RT was carried out with $500 \mathrm{ng}$ of total RNA from each sample using the RNA PCR kit (Promega, Madison, WI, USA). Target mRNAs analysed included those coding for P-gp and GAPDH. PCR products $(10 \mu \mathrm{l})$ were separated on 3\% agarose gels, and the gel imaging system (Vilber Lourma, Marne-la-Vallée, France) was used to scan the gel and quantify the levels of expression. Amplification of GAPDH was used as the control. As to the real-time quantitative PCR, All-in-One ${ }^{\mathrm{TM}}$ qPCR mix (GeneCopoeia, Rockville, MD, USA) and the primers for Sox 2, Oct4, c-Myc, Klf4, Nanog, and Bmi-1 were used to assess their relative mRNA quantities. The Roter-Gene software was 
employed to derive amplification and melting curves, and to calculate the ratios of expression of target mRNAs in hMDSCs compared with MOLT- 4 cells using the $2^{-\Delta \Delta C T}$ method.

Western blot analysis. MOLT4 cells and hMDSCs-MOLT4 were harvested and washed with PBS once and lysed in RIPA buffer. Protein content was then determined by the BCA assay. The extracted proteins were separated in a $12 \%$ SDS-polyacrylamide gel electrophoresis and transferred to PVDF membranes. The membranes were first blocked with $5 \%(\mathrm{w} / \mathrm{v})$ nonfat dry milk (NFDM) in TBST and then probed with the indicated primary antibodies with gentle shaking at $4{ }^{\circ} \mathrm{C}$ overnight. After washing three times ( 5 min per time), the membranes were incubated with the HRP-conjugated secondary antibodies for $2 \mathrm{~h}$. The signals were detected using an enhanced chemiluminescence detection kit (Thermo Scientific, Rockford, IL, USA). Western blot analysis was performed using the following antibodies: anti-Sox2 (R\&D Systems), anti-Oct4 (R\&D Systems), anti-cMyc (R\&D Systems), anti-Bmi-1 (R\&D Systems), anti-Klf4 (Abcam) and anti-Nanog (Abcam) antibodies, anti-GAPDH (Protech) and secondary antibodies were obtained from Guge Biology Company.

Measurement of cell proliferation by Cell Counting Kit- 8 $(C C K-8)$. Cells at $1.5 \times 10^{4} / \mathrm{ml}$ were added to 96 -well plates in $100 \mu \mathrm{l} /$ well. Each condition was run in 6 repeats and the plates were cultured in regular $\mathrm{CO}_{2}$ incubator. At 24,48 and $72 \mathrm{~h}, 10 \mu \mathrm{l}$ of CCK-8 solution was added to each well for an additional $3 \mathrm{~h}$ of culture before termination. Cell numbers were deduced by reading absorbance at $\mathrm{OD}_{450}$ on a microplate reader.

Tumor formation ability of MOLT-4 and hMDSCs-MOLT4 in vivo. SCID (severe combined immunodeficiency) mice were obtained from Huafukang Institute (Beijing, China) and raised in Individual Ventilated Cages in Wuhan University Center for Animal Experiment/Animal Biosafty Level III laboratory. Experiments using animal were approved by the Medical Ethics Committee of Wuhan University School of Medicine. Under sterile conditions, MOLT-4 cells and hMDSCs-MOLT4 cells were injected subcutaneously into the SCID mice $\left(1.5 \times 10^{6}\right.$ per mouse). Animals were observed and weighed, and tumors measured and weighed every other day. Tumor volume was estimated using the formula $\mathrm{V}=1 / 2$ (length $\mathrm{x}$ width ${ }^{2}$ ) $\mathrm{x}$ Mice were sacrificed after the tumors grew for 7 days, and tumor tissue sections were analyzed by hematoxylin and eosin $(\mathrm{H} \& \mathrm{E})$ and immunohistochemistry staining.

$H \& E$ and immunohistochemical staining. Tumor tissues from all xenografts were processed following the routine procedure after $24 \mathrm{~h}$ fixation. Tumor tissue sections were stained with H\&E. For immunohistochemistry, all samples were incubated at room temperature and washed with PBS. Tumor tissue sections of $4 \mu \mathrm{m}$ on glass slides were deparaffinized and rehydrated. Antigen retrieval was performed in a pressure cooker at $110^{\circ} \mathrm{C}$ for $5 \mathrm{~min}$ in retrieval buffer. Endogenous peroxidase activity was blocked with $3 \%$ hydrogen peroxide. Tumor tissue sections were then incubated with anti-CXCR4 or CD44 antibody (Abcam, USA) for $30 \mathrm{~min}$. Immunocomplexes were detected after incubation with HRP (horseradish peroxidase) polymer for $30 \mathrm{~min}$ followed by incubation with DAB plus substrate for $10 \mathrm{~min}$. Normal IgG from the same species of the primary antibody and diluted to match the concentration of the primary antibody was used as the negative control. Images were analyzed using Image-Pro Plus 6.0 software by obtaining positive integrated optical density (IOD. The average IOD value of all photos in each group was used to represent the IOD of the group and expressed as the mean \pm SD.

Statistical analysis. All experiments were repeated at least three times. Experimental data were expressed as mean $\pm \mathrm{SD}$, using SPSS17.0 statistical software for data analysis of variance (ANOVA) and Student's t-test. $\mathrm{P}<0.05$ was considered statistically significant.

\section{Results}

Response of three leukemia cell lines to doxorubicin (DOX). We first used MTT assays to evaluate the effects of DOX on three leukemia cell lines, i.e., HL60, K562, and MOLT4. We determined the $\mathrm{IC}_{50}$ of HL60, K562, and MOLT4 cells to DOX treatment to be $1.05,1.35$ and $0.6 \mu \mathrm{g} / \mathrm{ml}$, respectively. In all subsequent experiments, we used DOX at the above $\mathrm{IC}_{50}$ concentrations to treat cells over $48 \mathrm{~h}$ followed by collecting drug-surviving cells.

Analysis of CXCR4 expression in drug-surviving cells. CXCR4 and its ligand CXCL12 are highly expressed on acute promyelocytic leukemia stem cells and involved in regulating the migration of LSCs. We used flow cytometry (FCM) and immunofluorescence microscopy to assess the CXCR4 expression levels in HL60, K562, and MOLT4 cells and their drug-surviving cells (DSCs). There was no significant difference in CXCR4 expression between drug-surviving HL60 and K562 cells and their corresponding parental cells (Fig. 1A-C). However, compared with the parental cells, drug-surviving MOLT4 cells expressed much higher levels of the stem cell surface marker CXCR4 (45.8\% vs. 4.8\%; P<0.05; Fig. 1A and $\mathrm{C})$. In addition, we detected CXCR4 mRNA expression in MOLT4 cells and MOLT4 DSCs. The result showed increased CXCR4 mRNA expression in hMDSCs-MOLT4 vs. MOLT4 cells $(n=5, P<0.05)$ (Fig. 1D).

Stem cell-like properties of hMDSCs-MOLT4 cells. Next, we employed flow cytometry to examine the expression levels of several stem cell related markers including CD34, CD38, CXCR4, and CD44 in hMDSC-MOLT4 cells. The results showed that the expression levels of CD34 and CD38 on MOLT4 and hMDSC-MOLT4 cells were $0.2 \%$ vs. $1.7 \%$, and $0.3 \%$ vs. $2 \%$, respectively (Fig. 2), suggesting upregulation of both molecules in hMDSC-MOLT4 cells. CXCR4 expression went up significantly, from $0.5 \%$ in MOLT4 cells to $23.3 \%$ in hMDSC-MOLT4 cells ( $\mathrm{n}=5, \mathrm{P}<0.05)$ (Fig. 2$)$.

In contrast to the upregulation of the above three molecules, CD44, one of the cell adhesion molecule (CAM) family members associated with proliferation of some tumor cells (including multiple myeloma and AMl cells) and with drug resistance, did not show significant alterations. In fact, its expression showed a slight decrease, from $37.5 \%$ in MOLT4 cells to $30.3 \%$ in hMDSC-MOLT4 cells (Fig. 2). 
A
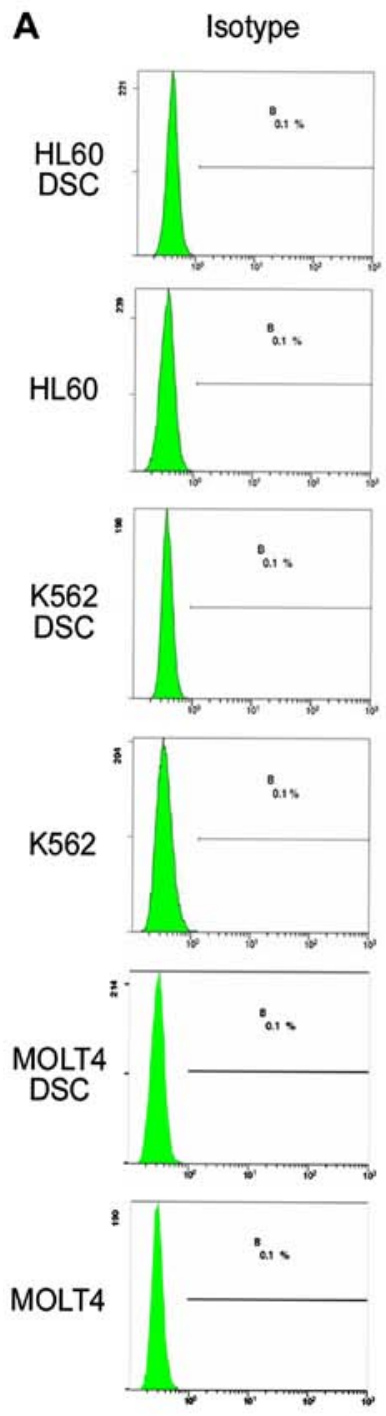

C

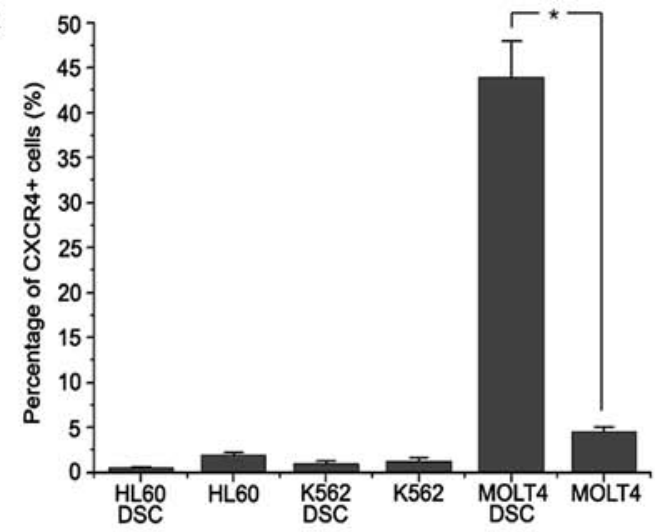

Experimental group
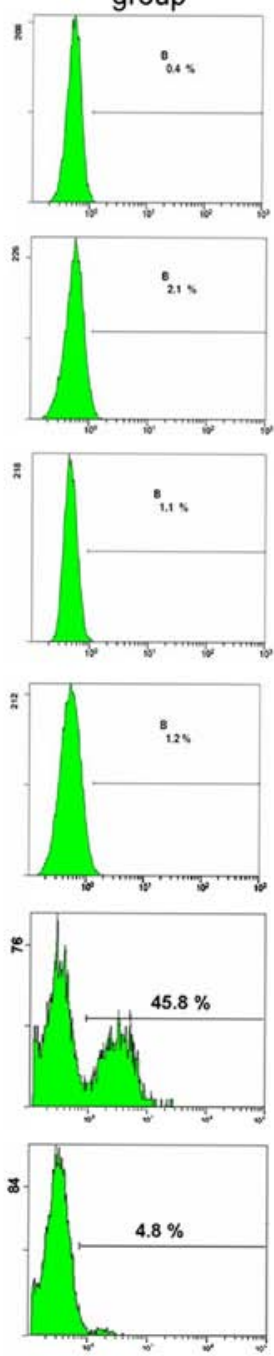

B
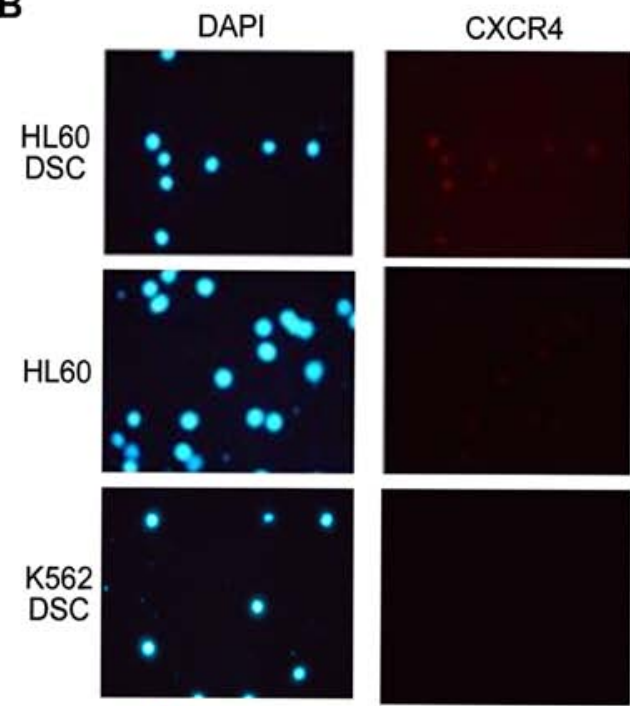

K562
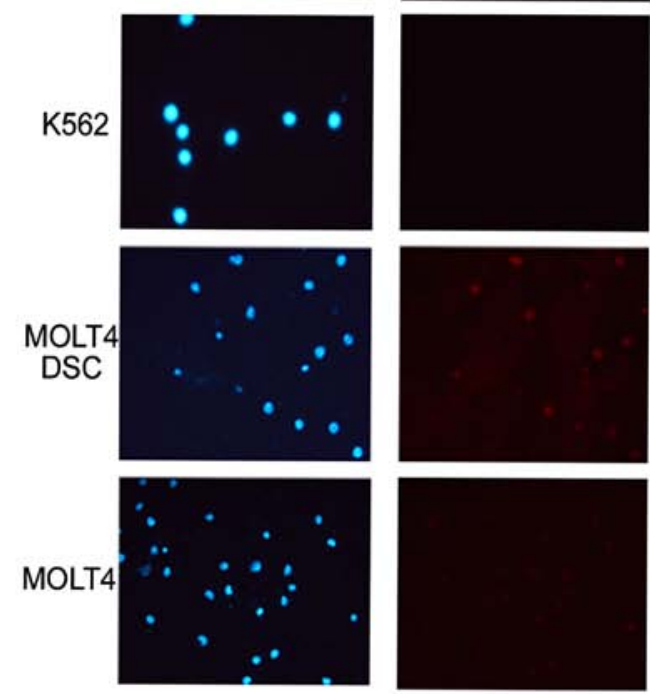

D

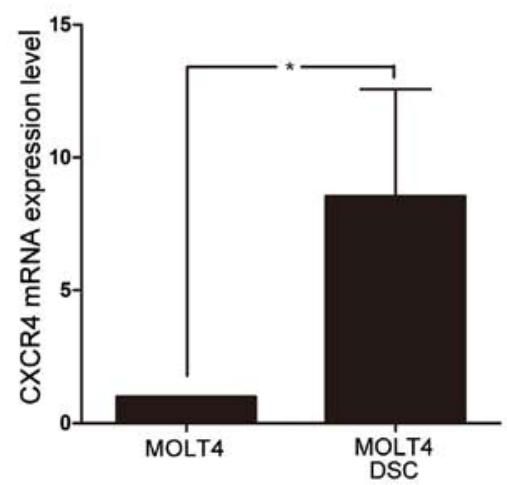

Figure 1. CXCR4 expression in drug-surviving leukemia cell lines. (A) Expression of CXCR4 in HL60, K562 and MOLT4 drug-surviving cells (DSC) and their parental cells stained with PE-anti-CXCR4 antibody and analyzed by FCM. (B) Expression of CXCR4 in cells stained with anti-CXCR4 antibody and analyzed by immunofluorescence. (C) Percentage of CXCR4 ${ }^{+}$cells in HL60, K562, and MOLT4 drug-surviving cells (DSCs) and their parental cells analyzed by FCM. $\left(\mathrm{n}=5\right.$; $\left.{ }^{\mathrm{P}}<0.05\right)$. (D) CXCR4 mRNA expression in MOLT4 cells and MOLT4 DSCs analyzed by real-time quantitative RT-PCR analysis. ( $\mathrm{n}=5$; $\left.{ }^{*} \mathrm{P}<0.05\right)$.

Increased drug resistance in hMDSCs-MOLT4 cells. $\mathrm{P}$-glycoprotein (P-gp) is one of the cell surface pump proteins that mediate drug resistance (24). We first used FCM to assess P-gp protein expression in MOLT4 and hMDSCs-MOLT4 cells. The results revealed increased P-gp in MOLT4 (3.5\%) vs. hMDSCs-MOLT4 (13.8\%) cells $(\mathrm{n}=6, \mathrm{P}<0.05)$ (Fig. 3A and B). Consistent with the FCM data, RT-PCR analysis also demonstrated increased P-gp mRNA expression in hMDSCsMOLT4 cells in comparison to MOLT4 cells (Fig. 3C). In addition, MTT assays showed that the $\mathrm{IC}_{50}$ values of parental 
A
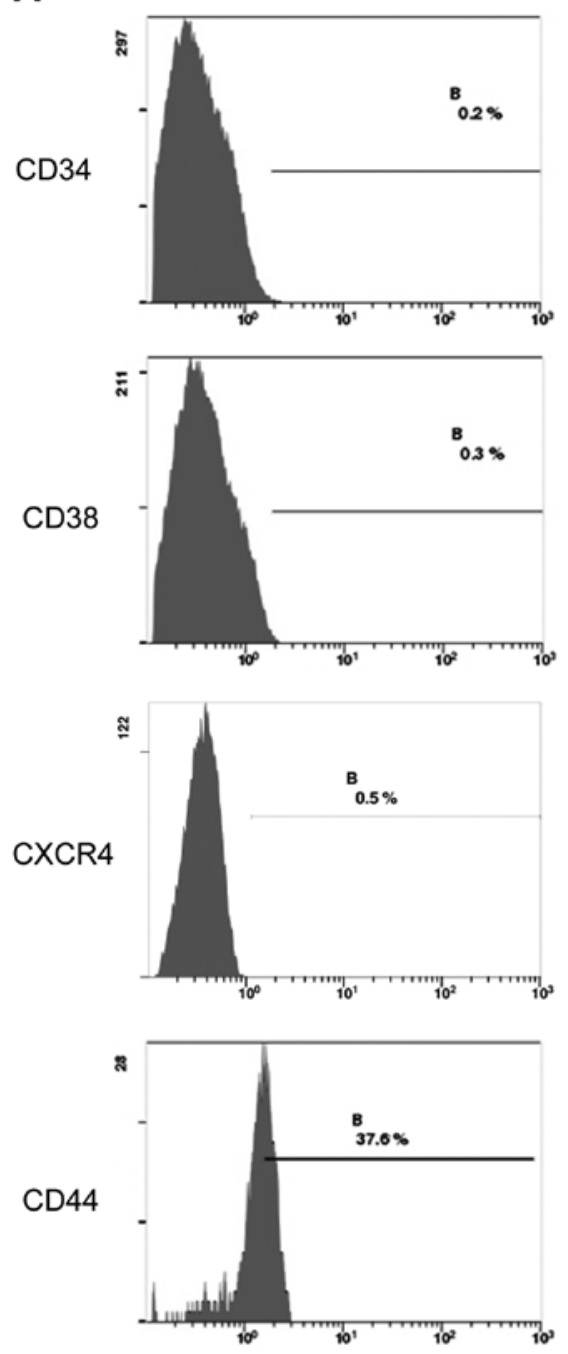
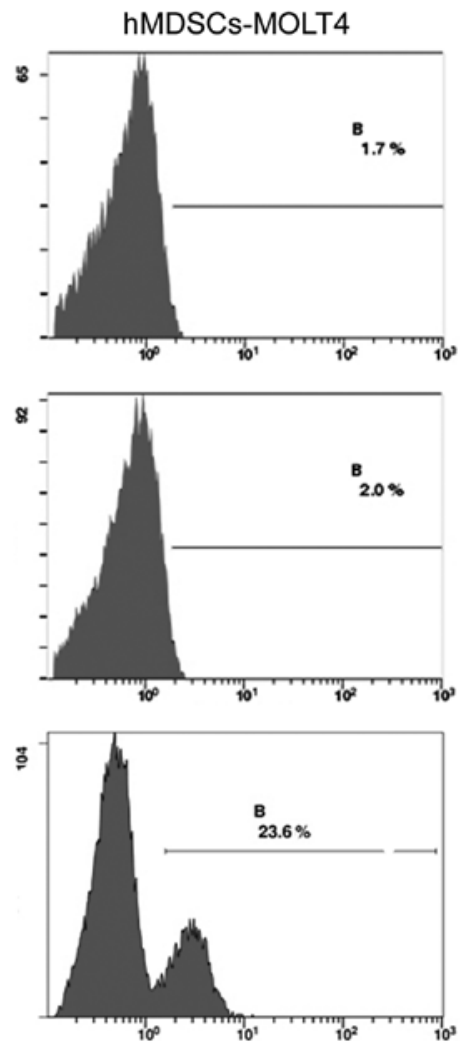

$\stackrel{2}{2}$

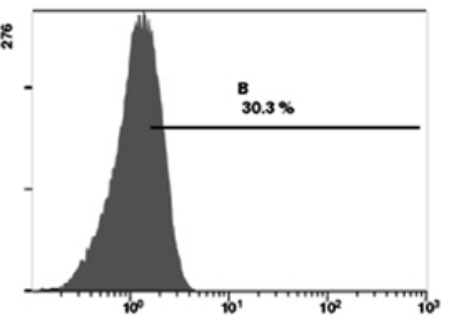

B

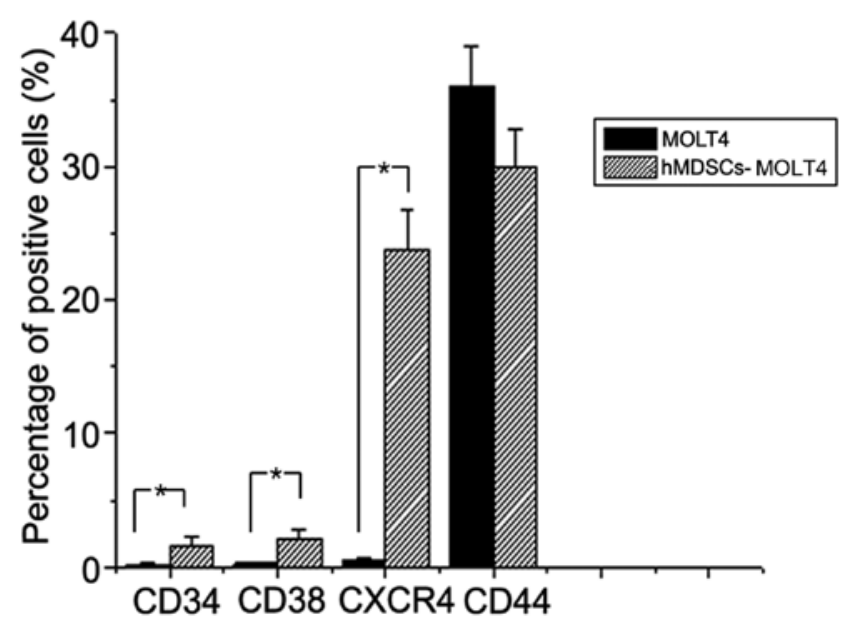

Figure 2. Expression levels of CD34, CD38, CXCR4 and CD44 in MOLT4 and hMDSCs-MOLT4 cells. (A) MOLT4 and hMDSCs-MOLT4 cells were stained with FITC-anti-CD34, FITC-anti-CD38, PE-anti-CXCR4, and PE-anti-CD44, respectively, followed by (B) FCM analysis. $\left(\mathrm{n}=5\right.$; $\left.{ }^{*} \mathrm{P}<0.05\right)$.

MOLT4 cells and hMDSCs-MOLT4 cells were 0.451 and 0.718 , respectively ( $n=6, P<0.05)$ (Fig. 3D), indicating enhanced drug resistance of hMDSCs-MOLT4 cells.

Increased expression of stem cell-related molecules in hMDSCs-MOLT4 cells. Subsequently, we carried out realtime quantitative RT-PCR and western blot analyses to assess the expression levels of Sox2, Oct4, c-Myc, Klf4, Nanog, and Bmi-1 in MOLT4 vs. hMDSCs-MOLT4 cells. The results revealed 2.5-, 5.4-, 4.3-, 3.1-, 3.6- and 4.1-fold higher mRNAs of Sox2, Oct4, c-Myc, Klf4, Nanog, and Bmi-1 in hMDSCsMOLT4 cells relative to those in MOLT4 cells (Fig. 4A). The protein level of Sox 2, Oct4, Klf4 and Nanog were also upregulated in hMDSCs-MOLT4 cells (Fig. 4B). 
A
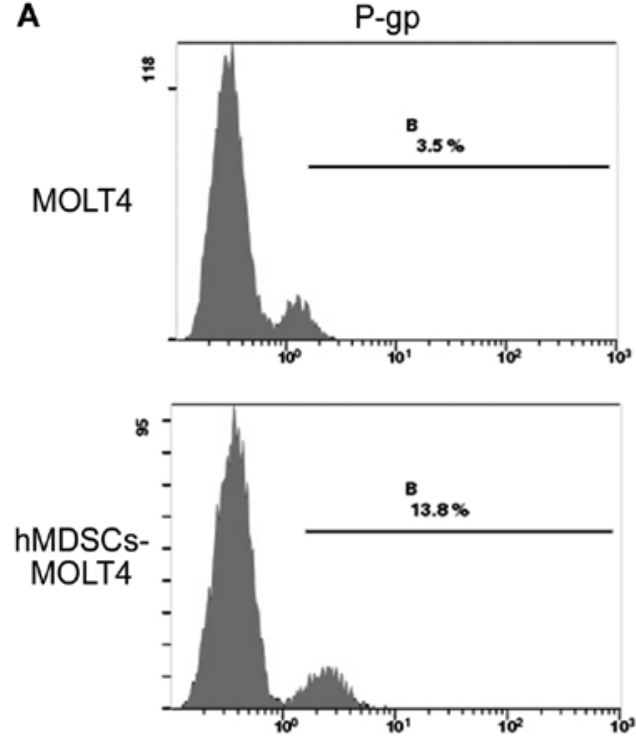

C

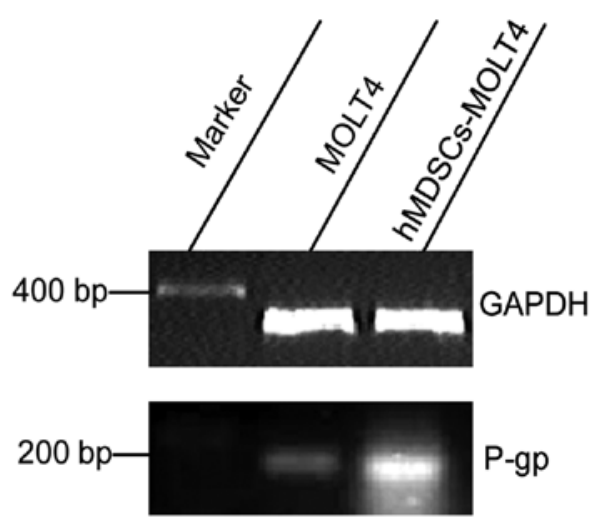

B

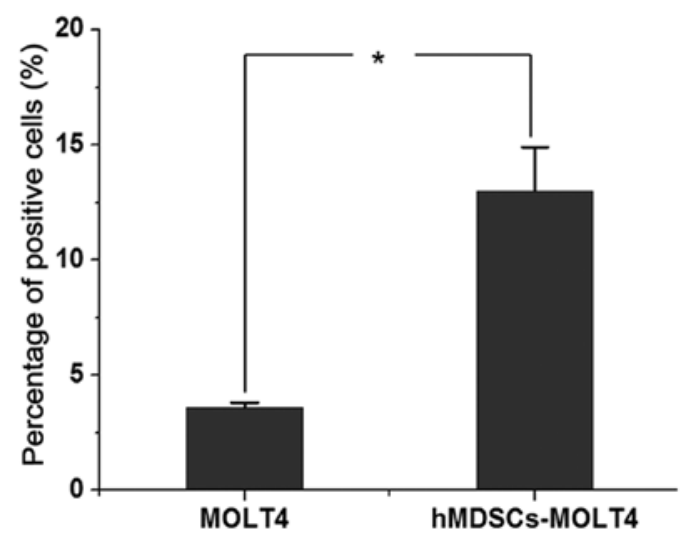

D

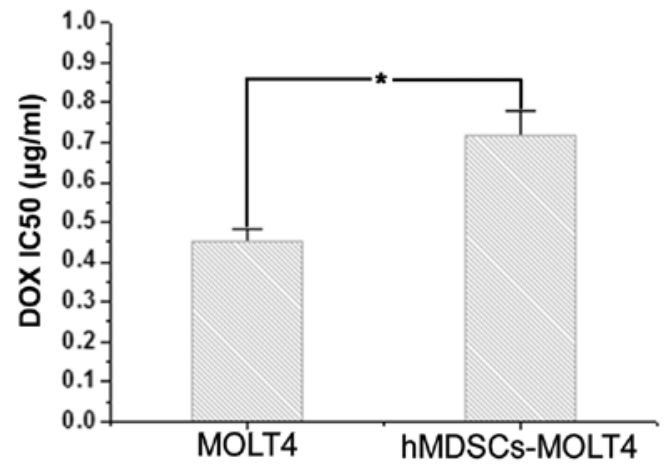

Figure 3. Drug resistance in hMDSCs-MOLT4 cells. (A) Expression of P-gp in MOLT4 and hMDSCs-MOLT4 cells stained with PE-anti-P-gp antibody and analyzed by FCM. (B) Percentage of positive cells $\left(\mathrm{P}_{-\mathrm{gp}}{ }^{+}\right.$cells) analyzed by FCM $\left(\mathrm{n}=6\right.$; $\left.{ }^{*} \mathrm{P}<0.05\right)$. (C) RT-PCR analysis of P-gp mRNA expression in MOLT4 and hMDSCs-MOLT4 cells. (D) $\mathrm{IC}_{50}$ values of MOLT4 and hMDSCs-MOLT4 cells as determined by MTT assays. Values are mean $\pm \mathrm{SD}$. ( $\mathrm{n}=6$; $\left.{ }^{*} \mathrm{P}<0.05\right)$.

\section{A}

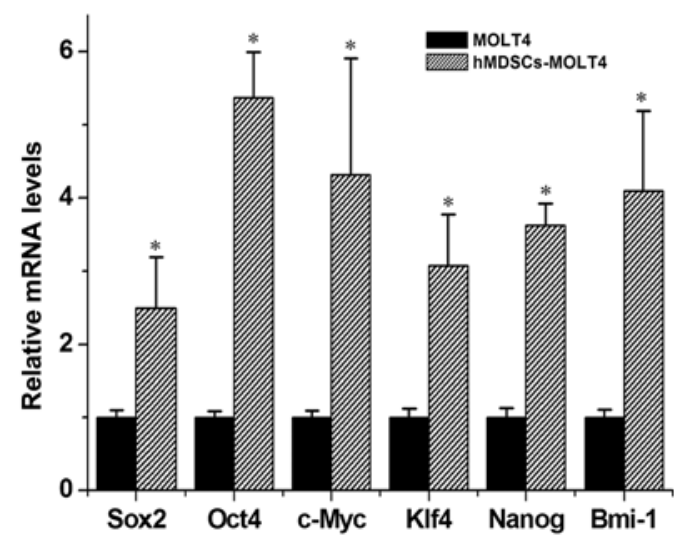

B

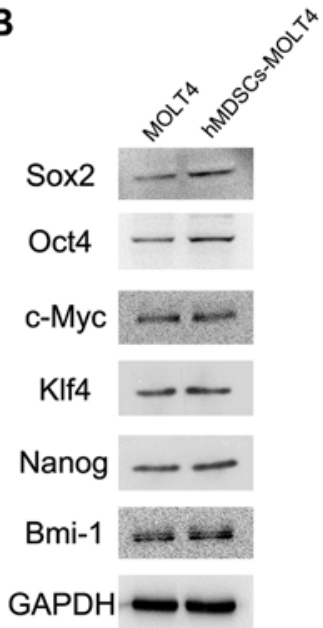

Figure 4. Expression of cancer stem cell related molecules in MOLT4 and hMDSCs-MOLT4 cells. (A) The mRNA expression levels of the indicated molecules in MOLT4 and hMDSCs-MOLT4 cells were determined by real-time quantitative RT-PCR analysis ( $\mathrm{n}=3$ ). GAPDH was used as internal reference for each sample, and the relative mRNA levels for each molecule in MOLT4 cells were set as one. "P<0.05, compared with MOLT4 cells. (B) Western blot analyses for indicated molecules in MOLT4 and hMDSCs-MOLT4 cells.

Decreased proliferation of hMDSCs-MOLT4 cells. We use the CCK8 assay to determine the relative cell proliferation. In this assay, CCK-8 is reduced to formazan by some intracellular dehydrogenase enzymes released by the mitochondria in 


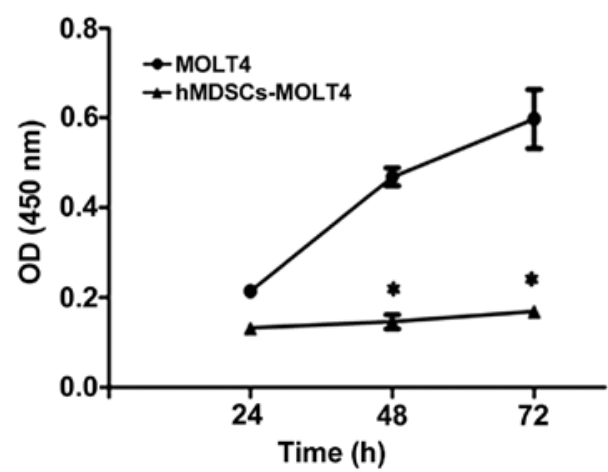

Figure 5. Proliferation of MOLT4 and hMDSCs-MOLT4 cells. Proliferation of MOLT4 and hMDSCs-MOLT4 cells was determined by CCK- 8 assays. Values represent the mean $\pm S D$. $\left(n=6 ;{ }^{*} P<0.05\right)$.

viable tumor cells. The $450-\mathrm{nm}$ absorbance is positively associated with the cell number. Our results revealed decreased proliferation in hMDSCs-MOLT4 cells in comparison to the parental cells (Fig. 5).

Tumorigenicity of MOLT4 and hMDSCs-MOLT4 cells. We then determined the tumorigenic potential of MOLT4 and hMDSCs-MOLT4 cells by injecting equal numbers of the two cell types subcutaneously in SCID mice. We did not observe any differences in the groups of mice with respect to hair coat, body weights and overall animal well being. However, the injected hMDSCs-MOLT4 cells developed much smaller and slower-growing tumors than MOLT4 cells (Fig. 6A). The group injected with MOLT4 cells developed tumors as early as 7 days post-injection whereas the group injected with hMDSCs-MOLT4 cells did not develop tumors until 17 days after tumor cell injections. Histological evaluations by $\mathrm{H} \& \mathrm{E}$ staining of tumor tissues indicated that the hMDSCs-MOLT4 tumors appeared to have more abundant connective tissues and blood vessels (i.e., tumor stroma) (Fig. 6B).

Finally, we analyzed, by IHC, the expression of CXCR4 and CD44 in tumor tissue sections. The results demonstrated that tumors derived from hMDSCs-MOLT4 cells showed significantly higher levels of CXCR4 $(n=9, P<0.01$; Fig. 6C and D) but slight lower levels of CD44 compared to tumors derived from MOLT4 cells (Fig. 6C and D).

\section{Discussion}

Some evidence has demonstrated that LSCs contribute to treatment failure. However, few studies have focused on the relationship of drug surviving leukemia cells and LSCs, not to mention the high migration drug surviving cells. Herein, we found that, compared with the parental cells, drug-surviving (short-term) MOLT4 cells expressed much higher levels of the stem cell surface marker CXCR4 which also related to the tumor metastasis. Then we have paid close attention to these hMDSCs-MOLT4 cells. For the first time, we explore stemness, drug resistance and metastasis in hMDSCs-MOLT4 cells.

It has been reported that CD34, CD44, Sox 2, Oct4, Nanog are the markers of cancer stem/progenitor cells and play an important role in maintaining their 'stemness' (25).
A

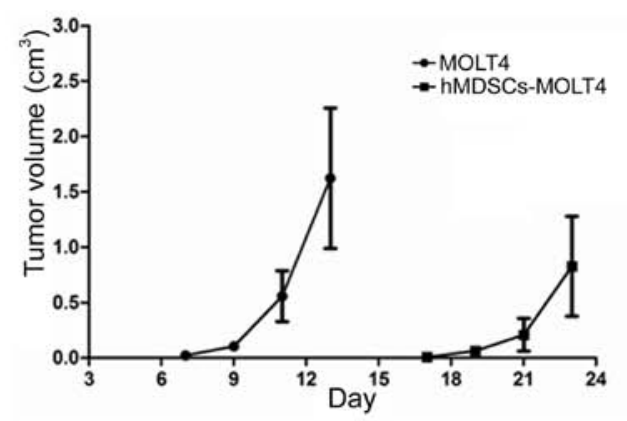

B

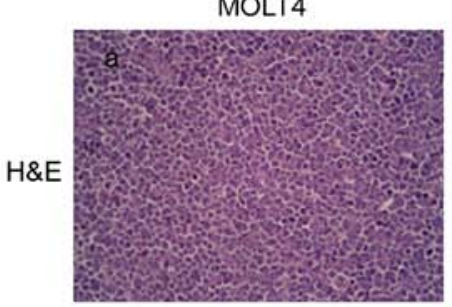

hMDSCs-MOLT4
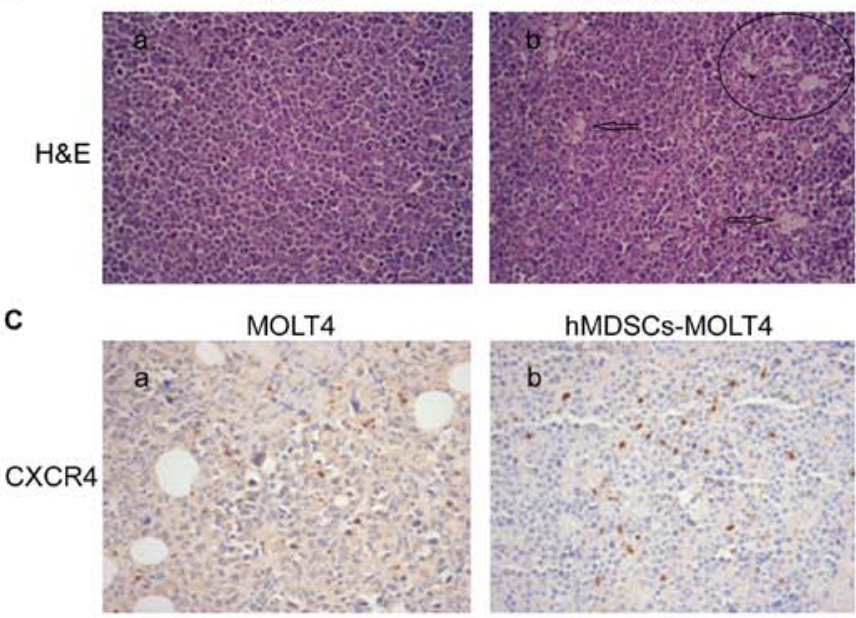

hMDSCs-MOLT4
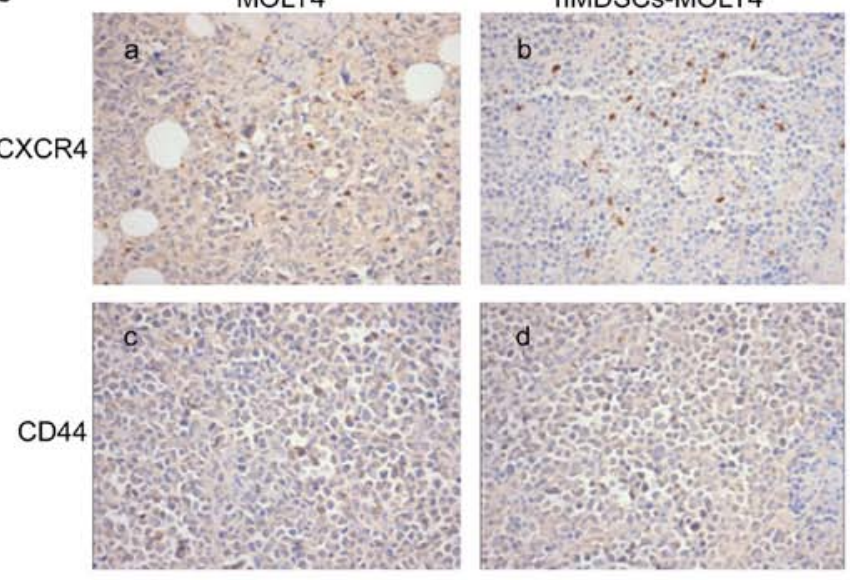

D

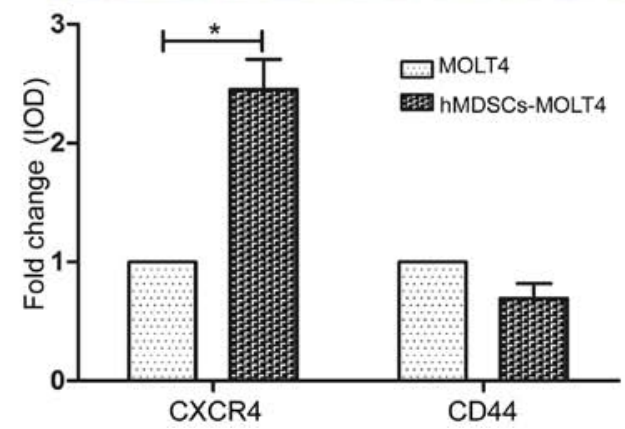

Figure 6. Tumorigenicity of MOLT4 and hMDSCs-MOLT4 cells and expression of CXCR4 and CD44 in tumor tissues. (A) Tumor growth curve MOLT4 and hMDSCs-MOLT4 cells injected in SCID mice. (B) H\&E staining of the tumor tissue section from SCID mice injected with MOLT4 and hMDSCsMOLT4 cells, respectively. (C) IHC analysis of CXCR4 and CD44 expression in tumors derived from MOLT4 and hMDSCs-MOLT4 cells, respectively. (D) Fold change (IOD values) of CXCR4 and CD44 in tumors, respectively $\left(\mathrm{n}=9 ;{ }^{*} \mathrm{P}<0.01\right)$.

C-Myc and KLF4 can maintain the stemness of stem cells and promote tumor formation (26). Bmi-1 is related to the self-renewal of leukemia stem cells (16). Another study showed that overexpression of C-Myc, Bmi-1, Oct4, Nanog in precancerous and cancerous cells may initiate oncogenic epithelial-mesenchymal transition and tumorigenesis, which plays important roles in the genesis of cancer stem cells (CSCs), malignant tumor initiation and progression, cancer 
metastasis, and drug resistance (27). In this study, hMDSCMOLT4 cells have some characteristics of leukemia stem-like cells with high expression of CD34, CXCR4, SOX2, OCT4, C-Myc, KLF4, Nanog and BMI-1.

The emergence of resistance to chemotherapy by tumor cells, when combined with metastasis, is the primary driver of mortality in cancer patients (28). The hMDSCs-MOLT4 cells, possessing some stemness of LSCs, highly expressed P-gp and demonstrated enhanced drug resistance of these cells. As is well known, metastasis is one of the primary biological characteritics of malignant tumors and the most important factor for the prognosis. We found that the expression of CXCR4, which is related to tumor cell homing and migration, was significantly higher in hMDSCs-MOLT4 cells than in MOLT4 cells. We also confirmed the high expression CXCR4 in tumor sections in vivo.

In addition, hMDSCs-MOLT4 cells seem to have a strong invasive potential in vivo, evidenced by strong interstitial and vascular tissues in tumor tissue sections. At the same time, the results showed that hMDSCs-MOLT4 cells exhibit decreased proliferation ability in vitro and in vivo. In line with our results, Stiehl et al reported that leukemia stem cells have a lower proliferative activity than other mitotic cell types and their replication is the rate-limiting process during expansion of leukemic cells. Moreover, slower cycling LSCs are the potential risk leading to relapse (29). Therefore, we speculate that hMDSCs-MOLT4 cells show similar characteristics with LSCs.

In this study, we also paid attention to another molecule, i.e., CD44. Researchers have reported that its high expression level is often related to proliferation and drug resistance in some tumor cells whereas some other studies presented opposite results $(22,23)$. We observed a slight decrease in CD44 expression in hMDSCs-MOLT4 cells in vitro and in vivo. In addition, we also observed that slightly decreased expression of CD44 was correlated with decreased proliferation of hMDSCs-MOLT4 cells in vivo and in vitro. Herein, we did not find the closed relationship between CD44 molecules and migration and drug resistance.

Prud'homme (30) suggests that CXCR4 and its ligand CXCL12 are metastasis-related key target molecules in the therapy of CSCs. Carter et al propose that imatinib combined with other drugs that target self-renewal and induce apoptosis could enhance the effect of imatinib on CMl cells (31). Our studies on hMDSCs-MOLT4 cells suggest that targeting stemness factors such as Oct4, Nanog, Sox2, Klf4 and CXCR4 may represent plausible options for eliminating T-ALL stem-like cells. Furthermore, an appropriate combination of drugs targeting the above mentioned stem cell factors and conventional chemotherapeutic agents may be a promising new treatment strategy. Finally, hMDSCs-MOLT4 cells may be used as a good research tool for finding novel drugs that could target cancer stem-like cells and prove to be effective in managing the relapse and metastasis of T-ALL.

In conclusion, the present study demonstrated that hMDSCs-MOLT4 cells exhibited strong drug resistance and certain cancer stem cell-like characteristics. For the first time, we proposed that stemness factors such as Sox2, Oct4, Klf4, Nanog and CXCR4 may be used as targets for eliminating T-ALL stem-like cells. It indicated that high migration drug surviving leukemia cells in T-ALL patients were likely to play a similar role to that of LSCs, and were probably important in the relapse of T-ALL. These findings provide new possibilities in understanding the relationship between high migration drug surviving leukemia cells and LSCs, and may present a new research direction for T-ALL relapse.

\section{Acknowledgements}

This work was supported by National Natural Science Foundation of China (no. 81400121, 81270607, 81541027) and Ph.D. Independent Research Program of Wuhan University (no. 410500106). We thank Ms. Weihuang Liu for FCM analysis.

\section{References}

1. Vyas P, Appelbaum FR and Craddock C: Allogeneic hematopoietic cell transplantation for acute myeloid leukemia. Biol Blood Marrow Transplant 21: 8-15, 2015.

2. Lang F, Wojcik B and Rieger MA: Stem cell hierarchy and clonal evolution in acute lymphoblastic leukemia. Stem Cells Int 2015: 137164, 2015.

3. Krause DS and Van Etten RA: Right on target: Eradicating leukemic stem cells. Trends Mol Med 13: 470-481, 2007.

4. Lane SW, Scadden DT and Gilliland DG: The leukemic stem cell niche: Current concepts and therapeutic opportunities. Blood 114: 1150-1157, 2009.

5. Schulenburg A, Blatt K, Cerny-Reiterer S, Sadovnik I, Herrmann H, Marian B, Grunt TW, Zielinski CC and Valent P: Cancer stem cells in basic science and in translational oncology: Can we translate into clinical application? J Hematol Oncol 8: $16,2015$.

6. Dallas NA, Xia L, Fan F, Gray MJ, Gaur P, van Buren G II, Samuel S, Kim MP, Lim SJ and Ellis LM: Chemoresistant colorectal cancer cells, the cancer stem cell phenotype, and increased sensitivity to insulin-like growth factor-I receptor inhibition. Cancer Res 69: 1951-1957, 2009.

7. Khan IN, Al-Karim S, Bora RS, Chaudhary AG and Saini KS: Cancer stem cells: A challenging paradigm for designing targeted drug therapies. Drug Discov Today 20: 1205-1216, 2015.

8. Li HZ, Yi TB and Wu ZY: Suspension culture combined with chemotherapeutic agents for sorting of breast cancer stem cells. BMC Cancer 8: 135, 2008.

9. Liu YM, Li XF, Liu H and Wu XL: Ultrasound-targeted microbubble destruction-mediated downregulation of CD133 inhibits epithelial-mesenchymal transition, stemness and migratory ability of liver cancer stem cells. Oncol Rep 34: 2977-2986, 2015.

10. Becker MW and Jordan CT: Leukemia stem cells in 2010: Current understanding and future directions. Blood Rev 25: 75-81, 2011.

11. Kornblau SM, Qutub A, Yao H, York H, Qiu YH, Graber D, Ravandi F, Cortes J, Andreeff M, Zhang N, et al: Proteomic profiling identifies distinct protein patterns in acute myelogenous leukemia $\mathrm{CD}^{2} 4^{+} \mathrm{CD} 38^{-}$stem-like cells. PLoS One 8: e78453, 2013.

12. Amaya $\mathrm{CN}$ and Bryan BA: Enrichment of the embryonic stem cell reprogramming factors Oct 4 , Nanog, Myc, and Sox 2 in benign and malignant vascular tumors. BMC Clin Pathol 15: 18, 2015.

13. Yin X, Zhang BH, Zheng SS, Gao DM, Qiu SJ, Wu WZ and Ren ZG: Coexpression of gene Oct4 and Nanog initiates stem cell characteristics in hepatocellular carcinoma and promotes epithelial-mesenchymal transition through activation of Stat3/ Snail signaling. J Hematol Oncol 8: 23, 2015.

14. Jeter CR, Liu B, Liu X, Chen X, Liu C, Calhoun-Davis T, Repass J, Zaehres H, Shen JJ and Tang DG: NANOG promotes cancer stem cell characteristics and prostate cancer resistance to androgen deprivation. Oncogene 30: 3833-3845, 2011.

15. Jeter CR, Badeaux M, Choy G, Chandra D, Patrawala L, Liu C, Calhoun-Davis T, Zaehres H, Daley GQ and Tang DG: Functional evidence that the self-renewal gene NANOG regulates human tumor development. Stem Cells 27: 993-1005, 2009.

16. Lessard J and Sauvageau G: Bmi-1 determines the proliferative capacity of normal and leukaemic stem cells. Nature 423: 255-260, 2003. 
17. Han AR, Lee JY, Kim HJ, min WS, Park G and Kim SH: A CXCR4 antagonist leads to tumor suppression by activation of immune cells in a leukemia-induced microenvironment. Oncol Rep 34: 2880-2888, 2015.

18. Dubrovska A, Elliott J, Salamone RJ, Telegeev GD Stakhovsky AE, Schepotin IB, Yan F, Wang Y, Bouchez LC, Kularatne SA, et al: CXCR4 expression in prostate cancer progenitor cells. PLoS One 7: e31226, 2012.

19. Dubrovska A, Hartung A, Bouchez LC, Walker JR, Reddy VA, Cho CY and Schultz PG: CXCR4 activation maintains a stem cell population in tamoxifen-resistant breast cancer cells through AhR signalling. Br J Cancer 107: 43-52, 2012.

20. Tavernier E, Aanei C, Solly F, Flandrin-Gresta P, Campos L and Guyotat D: CXCR4: A new therapeutic target of the leukaemic cell? Role of the SDF-1/CXCR4 axis in acute myeloid leukaemia Bull Cancer 101: 593-604, 2014 (In French).

21. Williams DA and Cancelas JA: Leukaemia: Niche retreats for stem cells. Nature 444: 827-828, 2006.

22. Liu C, Kelnar K, Liu B, Chen X, Calhoun-Davis T, Li H, Patrawala L, Yan H, Jeter C, Honorio S, et al: The microRNA miR-34a inhibits prostate cancer stem cells and metastasis by directly repressing CD44. Nat Med 17: 211-215, 2011.

23. Erb U, Megaptche AP, Gu X, Büchler MW and Zöller M: CD44 standard and CD44v10 isoform expression on leukemia cells distinctly influences niche embedding of hematopoietic stem cells. J Hematol Oncol 7: 29, 2014.

24. Assanhou AG, Li W, Zhang L, Xue L, Kong L, Sun H, Mo R and Zhang C: Reversal of multidrug resistance by co-delivery of paclitaxel and lonidamine using a TPGS and hyaluronic acid dual-functionalized liposome for cancer treatment. Biomaterials 73: 284-295, 2015.
25. Hämmerle B, Yañez Y, Palanca S, Cañete A, Burks DJ, Castel V and Font de Mora J: Targeting neuroblastoma stem cells with retinoic acid and proteasome inhibitor. PLoS One 8: e76761, 2013.

26. Wang X, Liu Q, Hou B, Zhang W, Yan M, Jia H, Li H, Yan D, Zheng F, Ding W, et al: Concomitant targeting of multiple key transcription factors effectively disrupts cancer stem cells enriched in side population of human pancreatic cancer cells. PLoS One 8: e73942, 2013.

27. Guo P, Gao A, Zhang G, Han $\mathrm{H}$ and Zhou Q: Decoding the knots of initiation of oncogenic epithelial-mesenchymal transition in tumor progression. Curr Cancer Drug Targets 13: 996-1011, 2013

28. Wu A, Loutherback K, Lambert G, Estévez-Salmerón L, Tlsty TD, Austin RH and Sturm JC: Cell motility and drug gradients in the emergence of resistance to chemotherapy. Proc Natl Acad Sci USA 110: 16103-16108, 2013.

29. Stiehl T, Baran N, Ho AD and Marciniak-Czochra A: Cell division patterns in acute myeloid leukemia stem-like cells determine clinical course: A model to predict patient survival. Cancer Res 75: 940-949, 2015.

30. Prud'homme GJ: Cancer stem cells and novel targets for antitumor strategies. Curr Pharm Des 18: 2838-2849, 2012.

31. Carter BZ, Mak DH, Cortes J and Andreeff M: The elusive chronic myeloid leukemia stem cell: Does it matter and how do we eliminate it? Semin Hematol 47: 362-370, 2010. 